\title{
Resolution Enhancement by Applying MFM Under UHV Conditions
}

\author{
Michael Dreyer, Romel D. Gomez, and Isaak D. Mayergoyz
}

\begin{abstract}
The enhancement in signal-to-noise ratio and lateral resolution in MFM in going from ambient pressure to UHV is demonstrated. The performance of several cantilevers is evaluated using a patterned $50 \mathrm{~nm}$ thick permalloy film, with cross-tie as well as $\sim 90^{\circ}$ domain walls, and a $200 \mathrm{~nm}$ thick permalloy film with perpendicular magnetization. The increase in the quality factor of the cantilever oscillation in UHV improves the sensitivity, consequently allowing less magnetic material on the tip to achieve the same signal-to-noise ratio. This reduction in magnetic volume sharpens the lateral resolution. We also demonstrate that the magnetic interaction can be so weak that a magnetic contrast is visible only under UHV conditions.
\end{abstract}

Index Terms-Magnetic force microscopy, resolution, UHV.

\section{INTRODUCTION}

$\mathbf{I}$ $\mathrm{N}$ THE past few years, magnetic force microscopy (MFM), applied under ambient conditions, has become a standard technique for measuring the magnetic stray field distribution of a magnetic sample. The advantages of this technique include high spatial resolution, as well as the possibility of measuring the topography of the same area on the sample surface. Unfortunately, the signal is proportional to the product of the probe moment and the sample field gradients so that it is impossible to simultaneously maximize the resolution and field sensitivity. High resolution demands a small magnetically active volume of the probe, which leads to low moments and consequently to less interaction. The "visibility" of magnetic features is therefore determined by the intrinsic signal-to-noise ratio $(\mathrm{S} / \mathrm{N})$.

Most varieties of MFM rely on the measurement of force gradients acting on the magnetic probe by measuring the frequency shift of the AFM cantilever. Early in the development of MFM, it was recognized that performing MFM in vacuum conditions should enhance the minimum detectable frequency shift, i.e., [1]:

$$
\left.\delta f\right|_{\min }=\sqrt{\frac{k_{B} T B f_{0}}{A^{2} c Q}}
$$

with Boltzmann's constant $k_{B}$, temperature $T$, bandwidth of measurement $B$, resonance frequency of the cantilever $f_{0}$, amplitude $A$ and spring constant $c$. The minimum detectable force gradient is given by $\left.(\partial F / \partial z)\right|_{\min } \approx\left(2 c \delta f / f_{0}\right)$. Since the shift in frequency $\delta f$ depends on the quality factor $Q$ of the resonance curve, the sensitivity should increase under ultra high vacuum conditions (UHV) as air damping is greatly reduced, besides

Manuscript received October 9, 1999.

The authors are with the Laboratory for Physical Science, College Park, MD 20740 USA (e-mail: dreyer@1ps.umd.edu).

Publisher Item Identifier S 0018-9464(00)08659-3. enabling the measurement of surfaces without protection/oxide layers, neither on the tip nor on the sample. This reduces the effective tip to sample distance. Despite the importance of high lateral resolution, the possible enhancement achievable in UHV has not yet been thoroughly studied. In this experiment, we systematically analyze this enhancement, and show that features virtually invisible in air can emerge in UHV.

\section{MEASUREMENTS}

We performed MFM using a commercial combined scanning force/scanning tunneling microscope (AFM/STM) operating in UHV [2]. In contact AFM mode the beam deflection method is used to detect the bending of the cantilever [3]. For noncontact AFM the cantilever is oscillated at its current resonance frequency by feeding the photodetector signal thru a phase shifter back to a piezo at the foot of the cantilever. The amplitude of the oscillation is kept constant and the frequency is measured by an FM-detector. The output signal of the FM-detector was amplified by a factor of 10 and subsequently low pass filtered with a cut off frequency of typically $60 \mathrm{~Hz}$.

Two permalloy films were used as samples. The films were deposited on silicon wafers with native oxide by thermal evaporation (base pressure $10^{-6} \mathrm{mbar}$, rate $10 \AA / \mathrm{s}$ ). The first film was patterned using lift off technique. It shows various bars and squares with lateral scales of $2.5-50 \mu \mathrm{m}$. The film had a thickness of $50 \mathrm{~nm}$, and the easy axis of magnetization was in the film plane. The size of the magnetic domains was in the range of $2 \mu \mathrm{m}$ to several $10 \mu \mathrm{m}$ depending on the structure. The domains were separated by Bloch- and cross-tie domain walls. At the edges of the structures, end domains with $\sim 90^{\circ}$ walls were commonly found. The second film was $200 \mathrm{~nm}$ thick. In this case the direction of the easy axis of the magnetization was perpendicular to the film surface. The film showed stripe domains of a width of $\sim 400 \mathrm{~nm}$.

Both samples were imaged in UHV $\left(\sim 10^{-11}\right.$ mbar $)$ using four different silicon cantilevers with spring constants of $2-8 \mathrm{~N} / \mathrm{m}$ and resonance frequencies in the range of $79-87 \mathrm{kHz}$. One cantilever was a commercial "high moment" MFM tip (labeled "DI Hi") [4]. The other three cantilevers were prepared within the same UHV system. "AC 2" and "AC 4" were coated with a 10-15 nm thick layer of iron, "AC 5" was dipped in an in situ prepared $14 \mathrm{~nm}$ thick cobalt film. After the measurements in UHV the system was vented with nitrogen gas and the same areas on the sample surface were measured for a second time. To compare two different cantilevers similar regions were scanned.

The $Q$-factors of the individual cantilevers were determined by measuring the amplitude response as a function of excitation 
TABLE I

VALUES OF THE $Q$-FACTORS OF FOUR CANTILEVERS MEASURED IN UHV AND AT AMBIENT PRESSURE

\begin{tabular}{l|c|c|c|c} 
Lever & $Q_{\mathrm{N}_{2}}$ & $Q_{\mathrm{UHV}}$ & $Q_{\mathrm{UHV}} / Q_{\mathrm{N}_{2}}$ & gain in $\delta f$ \\
\hline \hline AC 2 & 320 & 34059 & 106 & 10.3 \\
\hline AC 4 & 250 & 14504 & 58 & 7.6 \\
\hline AC 5 & 230 & 96953 & 422 & 20.5 \\
\hline DI Hi & 420 & 17993 & 43 & 6.5
\end{tabular}
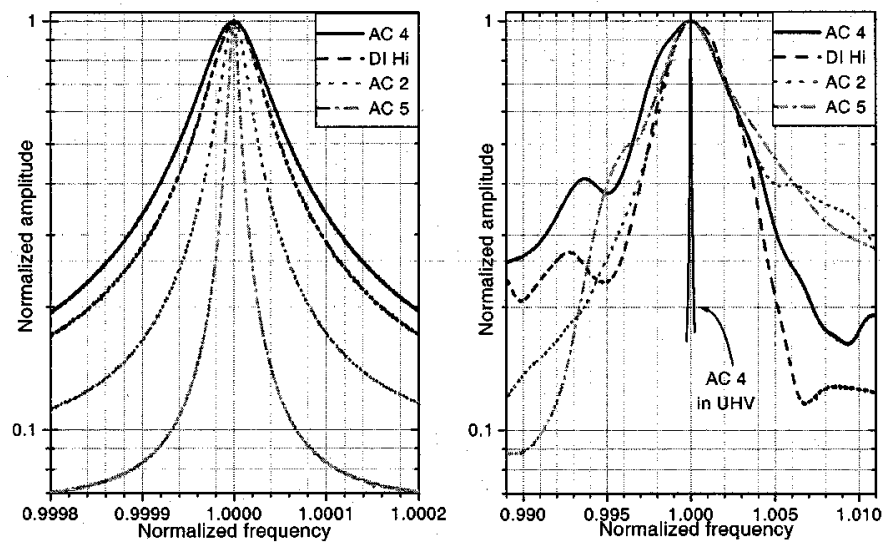

Fig. 1. Resonance curves measured for four cantilevers in UHV (left) and at ambient pressure (right). The estimated $Q$-factors are summarized in Table I.

frequency. The cantilever was excited by an external frequency generator (HP 33120A) and the amplitude of the oscillation, $A$, was measured at the output of the photodetector with a digital multimeter (Keithley). The excitation amplitude, $A_{0}$, was set so that $A$ at the resonance frequency was close to the amplitude during the MFM measurements. In UHV $A_{0}$ ranged from $0.5-5 \mathrm{mV}$, whereas at ambient pressure an amplitude of $120-800 \mathrm{mV}$ was needed. In UHV only the main resonance could be found, while at ambient pressure many sidebands could be observed which were unique to the respective cantilever.

\section{RESUlTS AND DISCUSSION}

To estimate the $Q$-factors of the cantilevers, the amplitude response function of a driven oscillator [1] in the presence of an independent noise source

$$
A(f)=\sqrt{a_{\text {noise }}^{2}+\frac{a_{0}^{2}}{\left(f^{2}-f_{0}^{2}\right)^{2}+\frac{f^{2} f_{0}^{2}}{Q^{2}}}}
$$

was fitted using a least $\chi^{2}$ method to the measured resonance curves. The results are summarized in Table I. The normalized resonance curves are displayed in Fig. 1. The $Q$-factors changed by a factor of 43-422 leading to an enhancement in $\mathrm{S} / \mathrm{N}$ of up to a factor of $6.5-20.5$.

Fig. 2 shows two examples of $\mathrm{S} / \mathrm{N}$ enhancement in MFM imaging using the high moment magnetic tip. The images (a) and (c) show measurements under UHV condition, while images (b) and (d) show the same location on the sample surface at ambient pressure. The images (a) and (b) show a corner of

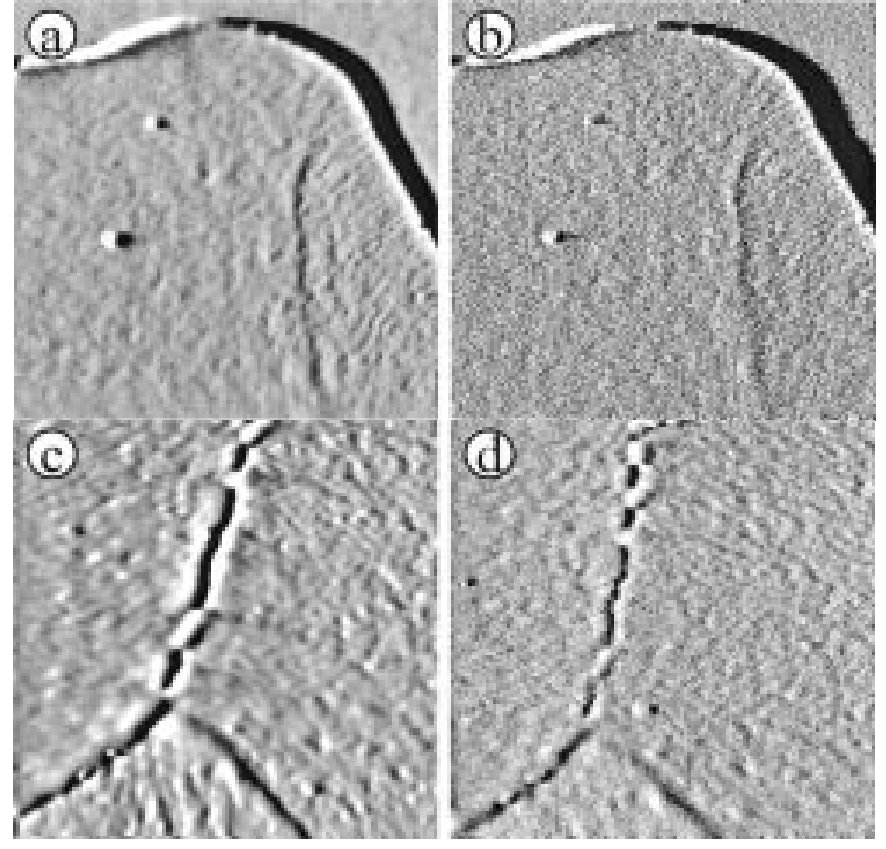

Fig. 2. Two examples of the enhanced signal-to-noise ratio on the $50 \mathrm{~nm}$ thick permalloy film. The image size is $3 \mu \mathrm{m} \times 3 \mu \mathrm{m}$ for images (a) and (b), $2 \mu \mathrm{m} \times$ $2 \mu \mathrm{m}$ for images (c) and (d), respectively. The images to the left were obtained in UHV, those to the right at atmospheric pressure. The difference in the noise level is evident.

the patterned permalloy film. The strong black and white contrast is due to topography and marks the step from the permalloy film down to the silicon oxide substrate. A single domain wall is ending in the corner by dissolving into a ripple structure [5]. Images (c) and (d) show a typical end domain configuration at the edge of a permalloy bar. The difference in noise level is evident in both cases. While under ambient conditions the domain walls could easily be seen, the magnetic ripple structure vanishes in the noise. Similarly, the walls are more diffuse under ambient conditions. Measuring the RMS noise on the silicon oxide of images (a) and (b) reveals a decrease in noise level by a factor of about 3. This value is about half the maximum possible gain of 6.5 (Table I), which could be explained by additional noise sources such as the electronics or a possible underestimation of the $Q$-factor at ambient pressure due to the sideband structure. Nevertheless, with increased S/N the MFM image is clearly enhanced in UHV.

The gain in sensitivity can be used to increase the lateral resolution. Reducing the amount of magnetic material on the tip, thereby reducing the tip radius, leads to an enhanced lateral resolution at the cost of magnetic sensitivity. The cantilever "AC 5 " gives a good example. The $Q$-factor changes by a factor of 422 leading to a maximum enhancement in resolution of 20.5. Fig. 3 shows a comparison to cantilever "DI Hi" using the $200 \mathrm{~nm}$ thick permalloy sample. Image (a) shows the magnetic contrast obtained with "DI Hi" in UHV. While the stripe domains are clearly visible with a high contrast corresponding to a frequency change of $21 \mathrm{~Hz}$, the transition between domains appears blurry. Cross sections show that the transition takes place within $100-80 \mathrm{~nm}$. Image (b) was obtained with cantilever "AC 5." The domain contrast averages to only $0.68 \mathrm{~Hz}$. Here, the transition length between two domains amounts to $\sim 40 \mathrm{~nm}$ 

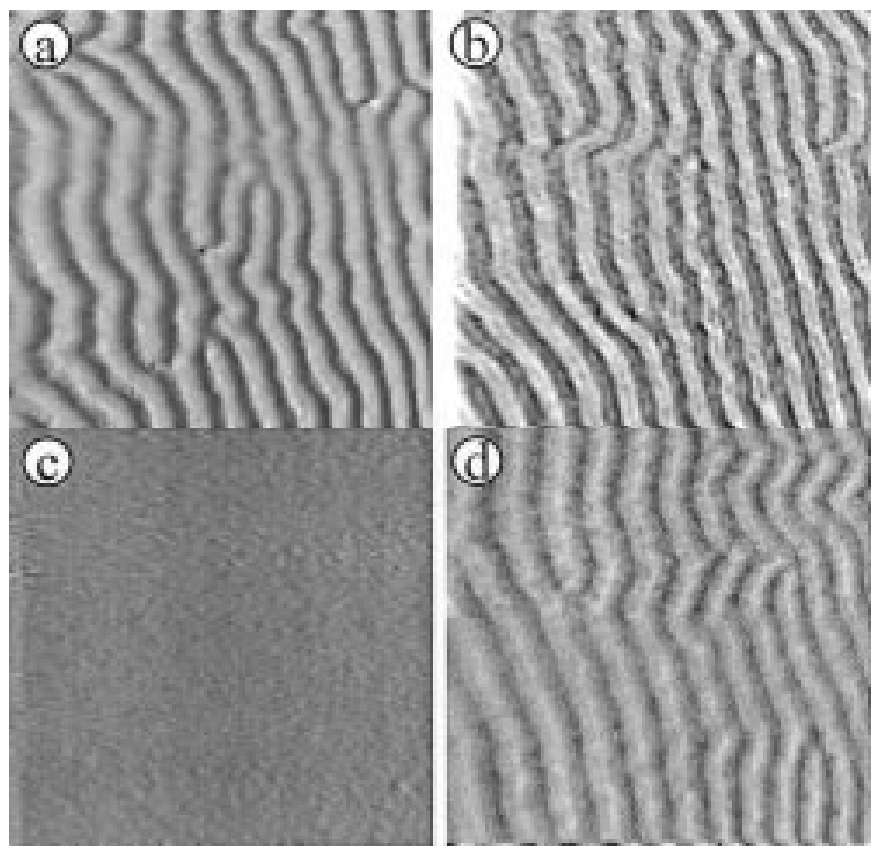

Fig. 3. Loss of magnetic contrast at atmospheric pressure demonstrated on the $200 \mathrm{~nm}$ thick permalloy film. Image (a) shows the magnetic domain structure measured with "DI Hi" in UHV. The black to white contrast equals $21 \mathrm{~Hz}$. Image (b)-(d) are measured with "AC 5." (b) UHV, contrast $0.68 \mathrm{~Hz}$. (c) $\mathrm{N}_{2}$, no magnetic contrast, (d) $\mathrm{HV}$, contrast $0.15 \mathrm{~Hz}$. The scan size is $4 \mu \mathrm{m} \times 4 \mu \mathrm{m}$ each.

leading to a visibly sharper image. The fine structure within the domains in image (b) can arise from topography or local magnetization variations. We are currently developing tools to distinguish between these possibilities.

Because of the low magnetic moment of the high resolution tip "AC 5," the drop in the $Q$-factor from UHV to ambient pressure leads to a loss of the magnetic contrast. Image c) in Fig. 3 represents the attempt to get a magnetic image. The scan rate was reduced to $1 / 16 \mathrm{~Hz}$, opposed to a typical scan rate of $1 / 4 \mathrm{~Hz}$, and the cutoff frequency of the low pass filter was set to $5 \mathrm{~Hz}$. Despite this effort, no magnetic contrast could be found. To exclude the possibility that the tip might have lost its magnetization, the chamber was subsequently pumped down to about $10^{-6} \mathrm{mBar}$, and the same area on the sample surface was scanned again using the normal scanning parameters. The result is displayed in image d) of Fig. 3. Despite the degraded contrast the magnetic features are again evident. The contrast degradation is not due to a reduction in $Q$-factor (here $Q \sim 88000$ ). Instead we believe that the residual $\mathrm{O}_{2}$ contents (partial pressure $<10^{-3} \mathrm{mBar}$ ) of the $\mathrm{N}_{2}$ gas degraded the magnetic moment of "AC 5." This finding indicates strongly that due to the increased $\mathrm{S} / \mathrm{N}$ found in UHV conditions much less magnetic material on the tip then in air is needed which increases the lateral resolution.

To demonstrate the important trade off between lateral resolution and S/N, the cantilevers "DI Hi" and "AC 5" were used

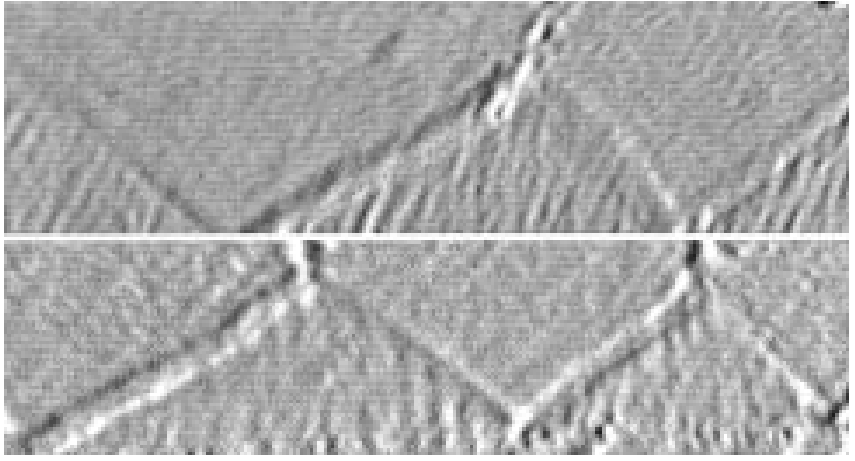

Fig. 4. Examples of the enhanced lateral resolution. The images show end domains on the $50 \mathrm{~nm}$ thick patterned permalloy film. Upper image: cantilever "DI Hi," magnetic ripple structure visible, wall thickness unclear, size: $2.25 \mu \mathrm{m} \times 0.63 \mu \mathrm{m}$, lower image: cantilever "AC 5," distinct difference in domain wall width, size $2.21 \mu \mathrm{m} \times 0.59 \mu \mathrm{m}$.

to take a closer look at the edge domains of the patterned $50 \mathrm{~nm}$ thick permalloy film in UHV. Two examples are displayed in Fig. 4. The edge of the patterned structure is in both cases at the lower end of the image. The upper image, measured using "DI Hi," shows the domain walls as well as a magnetic ripple structure. In comparison, cantilever "AC 5" shows an improved resolution at the domain walls, but the magnetic ripple structures are only barely visible. The magnetic interaction is too weak to image the magnetic ripples despite the increase in lateral resolution. Every second wall appears to be broader with a width $\sim 110 \mathrm{~nm}$ and $\sim 50 \mathrm{~nm}$ for the thinner wall. This is attributable to different wall angles. Since the direction of magnetization of the main domains have an angle of $\sim 60^{\circ}$ with respect to the side of the pattern, the wall angles are approximately $120^{\circ}$ and $60^{\circ}$. The ratio of the wall widths of 2.2 is in good agreement with the wall angle ratio of 2 .

\section{CONCLUSION}

The increased usage of MFM in UHV is imminent as we become more concerned with the micromagnetics of multilayer devices as they are fabricated. This work shows that UHV experiments are important not only in preventing surface contamination but also in significantly enhancing the sensitivity. Enhanced sensitivity further allows the reduction in the magnetic volume of the probe, which improves the spatial resolution. In this work we have demonstrated the feasibility of detecting weak magnetic features in UHV that would be invisible in air.

\section{REFERENCES}

[1] T. R. Albrecht, P. Grütter, D. Horne, and D. Rugar, J. Appl. Phys., vol. 69, p. $668,1991$.

[2] Idsteiner Str. 78 65 232“UHV STM/AFM," Omicron Vakuumphysik $\mathrm{GmbH}$, Taunusstein, Germany.

[3] G. Meyer and N. Amer, Appl. Phys. Lett., vol. 55, p. 1045, 1989.

[4] Nanosensors, High Moment Magnetic Cantilever.

[5] H. Hoffmann, J. Appl. Phys., vol. 35, p. 1790, 1964. 